https://doi.org/10.7203/Celestinesca.15.19749

\title{
DOS CONGRESOS EN 1991 CELEBRAN EL 450 ANIVERSARIO DE LA MUERTE DE FERNANDO DE ROJAS
}

\author{
Joseph T. Snow \\ Michigan State University
}

I. SALAMANCA, ESPAÑA, la semana del 26 al 30 de agosto. La octava semana de los "Cursos Intemacionales», dirigidos por Emilio de Miguel Martínez. El Curso Superior de Filología Hispánica, sesiones dedicadas al tema: "Revisión de la Celestina (en el $450^{\circ}$ aniversario de la muerte de Rojas)". Como ochenta inscripciones. Asi era el programa desarrollado:

Lunes, dia 26:

10-12. María Eugenia Lacarra (Univ. del País Vasco). "Humor y erotismo en la Celestina (I y II), día 26

12-14. Francisco Rico (Univ. Autónoma de Barcelona). "El problema estilístico de la Celestina" (I y II)

Día caluroso, con 80 asistentes en la Sala Unamuno del Edificio Central de la Universidad de Salamanca. Abre la sesión la catedrática de Literatura Medieval en Vitoria con un análisis de los focos de humor erótico en el primer auto de la obra, señalando la jocosidad que subvierte desde el comienzo el amor cortés, ideal de que se estaba mofando Fernando de Rojas. El humor mezclado con el erotismo es menos serio en el primer aucto (el que Rojas decía ser de otra mano que la suya) y la ponente dejaba para el segundo día una consideración de otros asuntos relacionados con este tema suyo.

El prof. Rico, en su turno, abrió su discusión sobre el problema estilístico con una consideración de la escena del conjuro de Plutón con que teimina el tercer auto de Celestina. Presentó la idea de los dos tipos de magia (por contacto y por analogia), afirmando que en los cuentos que nos contamos, o evitamos la realidad (analogía) o nos ahogamos en ella (contacto). Rojas, en su estilo, encuentra la manera (en la selección de los nombres, en el lugar 
o escenario de la acción, y más) de promover una recreación de la realidad 'naturalista' sin llegar a reproducirla. Siempre hay, en el realismo de Fernando de Rojas, un elemento artístico-literario que lo embellece. Así consigue romper la "rota virgili," mezclando el estilo sublime con el escandalosamente infimo. Rojas no encontraba modelos entre las otras eminencias de su época y tuvo a bien crearse su propio ideal, concretándolo en la Celestina.

Martes, día 27:

10-11. Prof. Rico. "El problema estilístico de la Celestina" (III) 11-12. Prof. Lacarra. "Humor y erotismo en la Celestina" (III)

Para acabar con su presentación, el prof. Rico escogió ilustrar con la lectura de y comentario a algunos pasajes de la obra: el de la imagen de Claudina presentada por Celestina a Sempronio (auto III): los devaneos de Calisto (auto VIII): y la entrada de Celestina en casa de Calisto (auto I). Subraya que el realismo especial de Rojas no es imitación de la realidad.

La profesora Lacarra ofreció abundantes ejemplos en el lenguaje de Rojas de humor y erotismo, utilizados en el arte de la caracterización (Elicia; Calisto, Pleberio), para demostrar que los "dichos lascivos, rientes" del texto demuestran un alto nivel de comprensión del sub-texto erótico aprovechado por Fernando de Rojas.

Miércoles, día 28:

10-12. Prof. Joseph T. Snow (Michigan State University, USA). "El arte de la caracterización en Celestina" (I y II)

12-13. Prof. Miguel Angel Pérez Priego (UNED, Madrid). "Presencia de la Celestina en el teatro renacentista" (I)

El prof. Snow quería en sus ponencias dejar. claro que la perfección de Celestina radicaba en buena parte en la creación de la sicología de los personajes, proyectada en el lenguaje que manipulan, unos en contacto con otros. Dedicaba esta sesión más bien al retrato de la evolución (doncella recatada a mujer libre) que experimenta Melibea en manos de Rojas, ofreciendo unos comentarios finales con respecto a Calisto.

Después, el prof. Pérez-Priego puso en relieve la resonancia de Celestina en el teatro del siglo XVI. Desde la "Egloga" de Pedro Manuel Ximénez de Urrea (1513) y pasando por Encina, Torres Naharro, se comienza a ver el perfil de esa resonancia celestinesca. Luego pasa a entrar en lo que se 
llamaría la comedia novelesca o "de enredo," tocando en nombres como Jaime de Huete, Luis de Miranda, y textos que han quedado en el anonimato ("Seraphina" por ejemplo). A partir de 1550 disminuyen las alusiones al enredo celestinesco (Lope de Rueda, Timoneda).

Jueves, día 29:

10-11. Prof. Snow. "El arte de la caracterización en Celestina" (III)

11-13. Prof. Pérez Priego. "Presencia de la Celestina en el teatro renacentista" (II y III)

Snow siguió desarrollando su tema con el contraste deliberado que hace Rojas entre Celestina y Pleberio, dos personajes íntimamente conectados en el esquema de la obra pero que nunca se encuentran en sus páginas. La 'batalla' entre ellos es dirigida por Celestina y el premio es el alma de Melibea. El fondo contra el cual tiene lugar esta desigual contienda se esboza en términos sociales, morales, y personales.

Pérez Priego siguió explicando la sombra de Celestina en la segunda mitad del s. XVI, haciendo hincapié en ciertos textos y haciendo a todos ver que era la figura de Celestina misma que atraía casi toda la atención de los dramaturgos de aquella época. Textos comentados incluían Plácida y Victoriano, la segunda Egloga de Urrea, la Comedia Tidea, el Auto de Clarindo, una farsa "de la Hechicera" por Diego Sánchez de Badajoz, y una farsa alegórica "de la Esposa de los Cantares," en la que la figura identificada con la alcahueta de Rojas lleva por nombre Ipocresía.

Viernes, día 30:

10-11. Joseph Pérez (Director de la Casa de Velázquez, Madrid). "La Celestina en la sociedad de su tiempo."

11-12. Jesús Puente (Actor y Director). "Para una representación actual de la Celestina."

Comenzó el prof. Pérez (el último Premio Nebrija) insistiendo en el marco histórico de Celestina, citando casos reales de excesos amorosos. Refleja, además, este texto la transición del mundo hispánico y europeo en el gusto por las novedades, la importancia del dinero y el comercio, y las nuevas relaciones dentro del marco urbano (con ejemplos de cada uno). Y terminó con el tema de Celestina como texto converso, diciendo que no le convencen los argumentos hasta ahora ofrecidos. No ve el menor indicio de origen judío, ni eco de las polémicas inquisitoriales: es más, no se saca nada claro sobre la situación de Fernando de Rojas del texto de Celestina. 
Jesús Puente habló de las excelentes cualidades teatrales de la Celestina y su propósito de colaborar con el prof. Emilio de Miguel en una representación integral de la obra en Salamanca, fuera de Salamanca, y fuera de España. El primer auto se representaria en una iglesia, habría pocos arreglos al texto para no perder las acciones psicológicas, se acentuaría más los 'silencio' del texto y se buscaria definir bien las personalidades (p. ej., Celestina no sería repulsiva: al contrario, siempre fue una cortesana, y sus artes lo reflejarian). El intento sería no aburrir con el montaje de la obra.

Como para dar cierre y fin a la última semana de estos Cursos Internacionales, ese mismo día aparecieron en el boletín del mismo organismo, Marcapasos, dos creaciones de tipo original relacionadas con Celestina. A continuación, la contribución (originalmente anónima) del Director mismo de los Cursos Internacionales, Emilio de Miguel, reproducida con su permiso:

\section{LA CELESTINA. Acto XXI}

(Manuscrito conservado en la redacción de Marcapasos)

[Emilio, Director de los Cursos Internacionales, tornando a su despacho con grandísimo llanto, pregúntale a Alberto, su colaborador en el regimiento del Curso Superior de Filología Hispánica, la causa del súbito mal que le aflige. Cuéntale éste la partida de todos los alumnos del Curso, mostrándole toda vacía el Aula Miguel de Unamuno.] Y HACIENDO SU PLANTO, CONCLUYE LA OBRA.

ALBERTO: ¿Qué es esto, señor Emilio? ¿Por qué son tus fuertes alaridos? Dime la causa de tus quejas. ¿Por qué maldices tu honrada vejez? ¿Por qué pides la muerte? ¿Por qué arrancas tus blancos cabellos? ¿Por qué hieres tu honrada cara? ¿Es algún mal del Curso Superior de Filología Hispánica? Por Dios, que me lo digas.

EMILIO: ¡Ay, ay, noble Alberto, nuestro gozo en el pozo; nuestro bien todo es perdido; no queramos más vivir! ¡Oh gentes que venís a mi dolor, oh amigos y señores, ayudadme a sentir mi pena! ¡Oh mis canas, salidas para sufrir pesar! Fálteme la vida, pues me faltó la agradable compañía de los inscritos en el Curso. ¡Oh duro corazón de Director! ¿Cómo no te quiebras de dolor, que ya quedas sin tus amados alumnos? ¿Para quién edifiqué la sede de los Cursos; para quién adquirí aparatos refrigeradores; para quién planté el césped del Patio de Escuelas Menores, para quién fabriqué las "aes"? ¡Oh, tierra dura! ¿cómo me sostienes? ¿Adónde hallará abrigo mi desconsolada vejez? ¡Oh vida de congojas llena, de miserias acompañada! ¡Oh mundo, 
mundo! Muchos mucho de ti dijeron, muchos en tus cualidades metieron la mano, a diversas cosas por oídas te compararon. Yo por triste experiencia lo contaré, como a quien las ventas y compras de tu engañosa feria no prósperamente sucedieron, como aquel que mucho ha hasta agora callado tus falsas propiedades por no encender con odio tu ira, porque no me. secases sin tiempo esta flor que este día echaste de tu poder.

Yo pensaba en mi más tierna edad que eras y eran tus hechos regidos por algún orden. Agora, visto el pro y la contra de tus bienandanzas, me pareces un laberinto de errores, un desierto espantoso, una morada de fieras, juego de hombres que andan en corro, laguna llena de cieno, región llena de espinas, monte alto, campo pedregoso, prado lleno de serpientes, huerto florido y sin fruto, fuente de cuidados, río de lágrimas, mar de miserias, trabajo sin provecho, dulce ponzoña, vana esperanza, falsa alegría, verdadero dolor. ¡Oh, incomparable pérdida, oh lastimado viejo, que cuanto más busco consuelos, menos razón hallo para me consolar!

¿Qué haré cuando entre en el Aula Miguel de Unamuno y la halle sola? ¿Qué haré de que no me respondan si les llamo? ¿Quién me podrá cubrir la gran falta que me hacen? Ninguno perdió lo que yo el día de hoy. Pues mundo halagüeño, ¿qué remedio das a mi fatigada vejez? ¿Cómo me mandas quedar en ti conociendo tus falsías, tus lazos, tus cadenas y redes, con que pescas nuestras flacas voluntades? ¿Quién acompañará mi desacompañada morada?

Del mundo me quejo porque en sí me crió, porque no me dando vida no engendrara en él al Curso Superior de Filología Hispánica. ¿Por qué te mostraste tan cruel con el viejo Director? ¿Por qué me dejaste, cuando yo te habia de dejar? ¿Por qué me dejaste penado? ¡Por qué me dejaste triste y solo in hac lachrymarum Valle?

$* * * * * 4 * * * * * * * * * 4 * 4$

J. T. Snow dio a conocer esta versión de un 'viejo romance' (descubierta la noche anterior en la posada de la Universidad). Se conoce bajo la rúbrica de «La muerte de Celestina».

Celestina nada teme, y en su casa duerme; no puede estar soñando que se acerca la muerte.

Sueña con su cadenita y con cient monedas de oro; no siente las pisadas; ni se entera del odio

que ya sombrea su puerta: su codicia no deja verlo. De esto Celestina nada presiente, ni se ha hecho 
nada para que no sea.

¡Tha! ¡Tha! ¡Tha! han dado tres

aldabonazos mortales:

Celestina no sabe qué es.

Se incorpora en la camà,

Celestina ya no duerme;

va $y$ abre a sus hijos,

abre $y$ hace que se entre

la muerte en dos espadas, en los ojos de sendas caras,

la labia le abandona

en la fría madrugada.
Celestina nada siente, y en su tumba duerme; otra cadena era la suya, se tejió en ella su suerte.

29-VIII-91.

\section{$+4$}

II. WEST LAFAYETTE, INDIANA (USA), los dias del 21 al 24 de noviembre de 1991. An International Conference in Commemoration of the $450^{\text {th }}$ Anniversary of the Death of Fernando de Rojas. Organizado por Ivy A. Corfis (Penn State University), J. T. Snow (Michigan State University) y Charles Ganelin (Purdue Univ.) Patrocinada por Purdue University, el Programa para la Cooperación Cultural España-Estados Unidos, la Sección Cultural de la Embajada de España en Washington D.C., y Celestinesca. Curiosamente, como en Salamanca, más o menos ochenta inscripciones.

\section{PROGRAMA DEL SIMPOSIO}

JUEVES, día 21. Llegada de los ponentes y asistentes. Una recepción en un restaurante local.

VIERNES, día 22. Bienvenida oficial de la oficina del Decano de la Purdue University.

10-11. Presentación por Ivy A. Corfis del plenarista ALAN D. DEYERMOND (Queen Mary-Westfield College--Univ. of London), quien disertó sobre el tema, "Female Societies in Celestina."

11-12:30. Sesión I.

Yakov Malkiel (Univ. de California, Berkeley): "An Analysis of Early Critical Reactions to María Rosa Lida de Malkiel's La originalidad artística de 'La 


\section{Celestina'.}

Emilio de Miguel Martínez (Univ. de Salamanca): "Celestina, teatro." (Defensa de la obra como concepción teatral)

Erna Berndt Kelley (Smith College): "Mute Commentaries of a Text: The Illustrations Discusión. of the Comedia de Calisto y Melibea." (Ilustrada)

13-14:30: Sesión II (traducciones; adaptaciones)

Theodore S. Beardsley, Jr. (The Hispanic Society of America): "The Latin Translation (1624) of Celestina by Kaspar von Barth."

Kathleen V. Kish (Univ. of North Carolina-Greensboro): "Eighteenth-Century Celestina Reincarnations." (Ilustrada)

Dwayne E. Carpenter (Boston College): "Celestina in Hebrew: A New Look at the Discusión.

First Hebrew Comedy."

16-17:30: Sesión III (teatro y teatralidad)

Joseph T. Snow. (Michigan State Univ.): "Five Centuries of Celestina Readings" (ilustrada con la "Celestina" de Serafín Estébanez Calderón)

Miguel Angel Pérez Priego (UNED-Madrid): "Celestina en escena: el impacto de la obra de Rojas en el teatro renacentista."

Adrienne Schizzano Mandel (California State Univ.-Northridge): "Celestina's Seductive Power in France." (Una lectura literario-musical de la ópera, Discusión.

"Célestine," de Maurice ỚHana)

SABADO, día 23

9:30-11:00. Sesión IV (estructuras sociales)

James F. Burke (Univ. de Toronto): "Law of the Father--Law of the Mother: Discourses of Power in Celestina"

Louise Fothergill-Payne (Univ. de Calgary): "'Conséjate con Séneca...': Auctoritas in Celestina and Celestina comentada."

Discusión.

11:30-13:00. Sesión V (caracterización)

Dorothy S. Severin (Univ. de Liverpool): "Celestina as a Comic Figure"

Jerry R. Rank (Saint Mary's College-Notre Dame): "'O cruel juez y que mal pago me has dado...'" (personajes productos del nuevo urbanismo)

Joseph V. Ricapito (Louisiana State Univ.): "People, Characters and Roles: A View of Characterization in Celestina." 
Discusión.

14:30-16:00. Sesión VI (ciencia y medicina)

Charles F. Fraker (Univ. de Michigan): "Celestina and the Four Temperaments."

Enrica J. Ardemagni (Indiana Univ.-Purdue Univ., Indianopolis): "Celestina's

Discusión

Laboratory: A Translator's Dilemma."

16:30-18:00. Sesión VII (leer-hablar in Celestina)

James R. Stamm (New York University): "Reading and Listening in Celestina."

Edward H. Friedman (Indiana University): "Rhetoric at Work: Celestina, Trotaconventos, and the Persuasive Arts."

Discusión.

19:00-22:00. BANQUETE (Morris Bryan Restaurant). Después de la cena, J. T. Snow presentó a MARIA EUGENIA LACARRA (Univ. del País Vasco) quien leyó la segunda plenaria, "La evolución del reglamento de la prostitución en la Castilla del siglo XV y la mancebía de Salamanca en tiempos de Fernando de Rojas."

Después (ver abajo), hubo algunas presentaciones humorísticas a cargo de los tres hispanomedievalistas procedentes del Reino Unido (una sorpresa para el "senado"). Se nos ha dado permiso para la publicación de dos de las presentaciones.

DOMINGO, día 24.

9:30-11. Sesión VIII (herencia de Celestina)

Nicholas G. Round (Univ. de Glasgow): "Celestina, Aucto I: A Platonic Echo and its Resonances."

Nicasio Salvador Miguel (Univ. Complutense-Madrid): "El rocho de Celestina en la Discusión. literatura española."

11-12:30. MESA REDONDA. Participaron María Eugenia Lacarra (moderadora), Alan Deyermond, Dorothy S. Severin, y Louise Fothergill-Payne. Los cuatro lograron resumir el trabajo del congreso, pasando revista de todas las aportaciones y dejando cerrado el congreso con una síntesis global de su contenido.

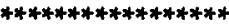

He aquí dos de los 'postres' que se sirvieron a los comensales en el banquete del congreso. El primero es obra de Alan Deyermond y él mismo, acompañado de un coro de doce posgraduados de la Miami University (Oxford, Ohio: alumnos de la 
prof. Constance Wilkins en un curso-seminario sobre Celestina), lo cantaron: la música era de una barcarola inglesa.

I'll sing you a song of an old Spanish text,

Celestina,

And soon you'll be saying, "Well, whatever next?"

For the story it gets no cleaner.

'Tis the tale of a youth that got badder and badder,

Till he met his deserts and fell off a ladder,

At the which his girlfriend, she looked very sour,

And she grumbled at Dad, and she jumped off a tower.

Why, thank you, sirs and ladies,

You ask for some more?

You've had Celestina

Here's Libro de buen amor.

This book it was written by a certain Archpriest,

And truth for to tell, he was worse than a beast.

His hero at first seem scared of serranas,

But with Dona Endrina he just went bananas.

At first, the hero seems much over-sexed,

But are you quite sure that you've read the subtext?

Any reading of the book must still be precarious,

So we'll just have to wait for vol. III of Zahareas.

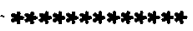

El segundo "postre" era uno muy rico, presentado y cantado por Nicholas G. Round, de Escocia. Es traducción de un fragmento del texto original versificado de Celestina, y se canta así:

There once was a lad named Calixtus

Who for reasons I dont understand

Set out to pursue amor mixtus

On his horse with his hawk in his hand.

When he met with the fair Melibea,

He hailed her in terms that were bold:

As his god, his platonic idea

But the shoulder she gave him was cold. 
Back home with his servant Sempronio, He shut himself up in his room, and there in the dark, on his own-io went in for some serious gloom. ?

Perceiving a chance of some profit, His lackey contested this mood; And incited the boss to come off it, For reasons both lucid and lewd.

And to set all to rights, this same joker Proposed they bring into the game An erotic consultant and broker Of note-Celestina by name.

'Do that,' says Calisto, 'instanter, And promise her money to boot.' So Sempronio set off at a canter: To her house (of uncertain repute),

Where his girlfriend was nearly discovered, Entertaining a guest (Crito) whom She promptly shoved into a cupboard, And he stayed there, disguised as a broom.

'There's work to be done,' says the messenger. Celestina: 'so put on your hat And leave this young woman here, blessing her, To lock up and to put out the cat.'

They returned to Calisto's fine mansion And, as their approach he descried, In a mood of ebullient expansion, He bade Pármeno bring them inside.

'That ratbag! That ancient obscenity!' Said the pageboy: 'I know her of old! If you value your life and amenity, You'll leave her out there in the cold.' 
'She's my auntie, you insolent puppy!'

Calisto exploded in rage.

'Stop standing agape like a guppy,

And show some respect for old age!'

So suiting the deed to the notion,

Calisto throws open his door

And displays his respectful emotion

By grovelling 'round on the floor.

'Calisto, fear not,' said the bawd; 'it Shall be as you wish with this dame -Provided that you can afford it (If not, then I'm sorry I came).'

Says Calisto: 'That's not the position. You're my hope, my salvation, my solace!

So there'll now be a brief intermission Whilst I zip upstairs for some dollars.'

Celestina was left there with Pármeno Thinking: 'How to get shot of this bum? It should make him less willing to harm me, no? If I tell him some tales of his mum.'

So she told of Claudina's activities In terms that would make your hair curl, And she flattered his sinful proclivities, By promising gold and a girl.

Till, with Pármeno quite despistado,

Calisto came hurrying back, To reward Celestina's bravado With a great deal of cash in a sack.

That makes seventeen verses (in dactyls) And I think its becoming a bore. It isn't as though the world lacked ills! So I'll stop. El antiguo Autor.

24-XI-91 Nicholas G. Round 


\title{
An International Conference in Commemoration of the 450th Anniversary of the Death of Fernando de Rojas
}

\subsection{November 1991}

\author{
Purdue University \\ West Lafayette, Indiana
}

\section{Sponsored by}

\author{
Purdue University \\ School of Liberal Arts \\ Department of Foreign Languages and Literanures \\ International Programs, Global Initiatives Grant Program \\ Program for Cultural Cooperation Between Spain's \\ Ministry of Culture and United States' Universities \\ Embajada de España, Oficina Cultural
}

\section{Celestinesca}

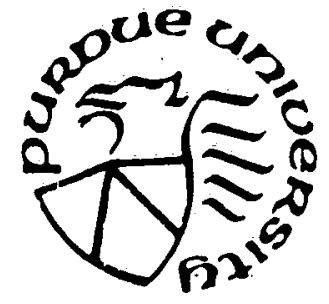

